\title{
Hemodialysis Canula Insertion- Ultrasound and Seeker Needle Method Revisited
}

\author{
Author \\ Rohit Herekar \\ Department of Medicine Government Medical College Miraj \\ Corresponding Author \\ Dr Rohit Herekar \\ Assistant Professor GMC Miraj, India \\ Email:drrohitherekar@gmail.com,8605512787
}

\begin{abstract}
The insertion of Hemodialysis canula is the first step towards doing hemodialysis. The Internal jugular vein is a common site for canulation and insersion is done by landmark method and Seldinger wire technique. The insertion of HD canula by ultrasound guidance is considered standard for central line insertion but seeker needle method in landmark technique can be used where ultrasound cannot be feasible. In this article we discuss about Seeker needle method and its advantages and disadvantages with Ultrasound method.
\end{abstract}

Keywords- Seeker needle, Hemodialysis canula, internal jugular vein, Landmark technique.

\section{Introduction}

The central venous placement of catheter is commonly performed in ICU settings. The use of central lines is increased significantly in ICU settings. ${ }^{1}$ The same principle of central ine insertion is used for Hemodialysis canula which is the first step in performing hemodialysis. The internal jugular vein (IJV) is the most common site for cannulation for hemodialysis canula ${ }^{2}$. The indication for inserting hemodialysis canula is to provide a temporary high flow acsess to Hemodialysis. Hemodialysis is an essential life saving procedure serving as renal replacement therapy to remove fluid and waste products in patients of acute and chronic renal failure.

The procedure and technique very similar to a central venous catheter insertion. Whilst the insertion is beneficial in critical cases inserting the catheter risks arterial puncture, pneumothorax, and other complications and correct technique should be used to save time and money. ${ }^{3}$

Three central veins typically used for venous access are Internal jugular Vein, Subclavian Vein, Femoral vein. The cannulation of internal jugular vein is important skill the common approaches being central and posterior. The right internal jugular vein in direct line with superior vena, absence of thoracic duct and ease of insertion for right handed operator makes it preferable for HD canula insertion which is done by Seldinger wire technique. ${ }^{4}$

A learning curve exists for central venous access procedures. Experienced operators enjoy greater success rates with fewer complications ${ }^{5}$. Among experienced and inexperienced operators, the 
number of needle passes correlates with the complication rate, rising significantly following two to three unsuccessful passes. ${ }^{6}$

The Ultrasound technique is recommended for insertion of HD canula. However needs availability of operator and machine and its universal use is still not followed in adults. ${ }^{7}$ In this article we discuss use of Seeker needle method of Landmark technique and its benefits and its advantages or disadvantages over other methods

\section{Landmark Technique: ${ }^{8}$}

The patient is placed in supine or tredelenberg position, draping and sterile environment is maintained to minimize infective complications. Slight head rotation 30 degrees to left for right IJV canulation. Then coats of sterilsing solutions like spirit and betadine to larger area near internal jugular vein. The Hemodialysis canula set assembly is kept open . (Fig.1) A $25 \mathrm{G}$ needle is used to give lignocaine subcutaneusly over large area of IJV.

In conventional method the large $16 \mathrm{G}$ needle is directly inserted palpating the carotid artery medially. In the apex of triangle formed by sternal and clavicular heads of sternocleidomastoid and clavicle inferiorlyat an angle of 45 degress. Continuous monitoring of pulse and saturation is carried out during procedure.

\section{Seeker Neddle}

Before inserting the HD canula needle of $16 \mathrm{G}$ the

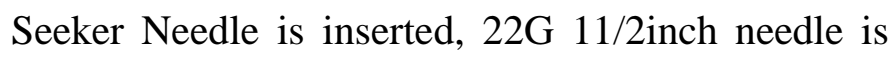
used, the carotid artery is palpated medially, at an angle of 30-45 degrees directing towards ipsilateral nipple the seeker needle inserted in skin and then gradually apply continuous negative pressure by pulling back on the plunger during advancement and withdrawal. Vessel penetration will go unrecognized unless negative pressure is applied. ${ }^{9}$ Confirm an intraluminal position by visualizing the steady flow of dark blood into the syringe. Bright red or high pressure pulsatile blood are important but imperfect clues to arterial puncture advancing and aspirating till blood flow reached. ${ }^{10}$ Remove assembly if bright colour pulsatile blood comes and try again checking landmarks. The needle is advanced and withdrawn in same vector to minimise lacerations to surrounding area. The vein will be reached at around $1-3 \mathrm{~cm}$ from skin.

Following the localisation the large HD canula needle can be inserted taking help of direction and depth of Seeker needle and rest canula can be inserted using Seldinger wire technique. ${ }^{11}$

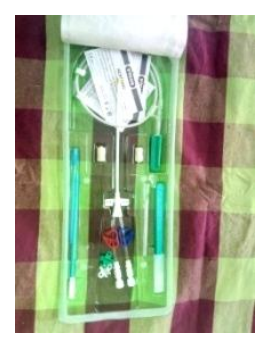

Fig. 1. Hemodialysis canula pack

\section{Ultrasound Technique}

The Ultrasound guided Hemodialysis canula insertion by dynamic method is the ideal method for insertion. The high frequency linear transducer 6-10 $\mathrm{MHz}$ is used. The radiologist is in ipsilateral view and assistant at the head end of patient. Using sterile covering and sterile jelly the internal jugular vein is identified. The views used are long axis view and short axis view the vein is identified by the phasic respiratory pulsations or by compressibility or by valsalva manoeuvre.

The inserting needle tip located in longitudinal view and once entered in vein flash appears and further guide wire $\mathrm{J}$ tip can also be identified. rest procedure can be done by needle over wire method. The Ultrasound guided method can be combined with seeker needle before insertion of HD canula needle. The ultrasound guidance is helpful for patients with difficult sticks, difficult anatomy, short neck, morbid obesity.

\section{Pros and Cons}

There is increased used of ultrasound in acute clinical care setting.12. The anatomy of the internal jugular vein was sufficiently aberrant in $8.5 \%$ of cases to complicate access by a landmark method, and five patients $(2.5 \%)$ had thrombosed internal jugular veins. ${ }^{13}$ 
The ultrasound guidance method has shown to reduce complication and is now recommended method for any central line insertion. Not until 1986 was the use of real-time ultrasonographic guidance for IJV cannulation reported. Various organisation support use of Ultrasound as efficient and core need to ICU central venous line insertion. ${ }^{14}$ With the use of landmark technique complications as high as $30 \%$ are seen. ${ }^{15}$

Some advantages of Ultrasound over landmark techniques include

- exact anatomy identified

- multiple pricks avoided

- reduced failure rate

- reduced sepsis

- useful in difficult sticks

- less time for procedure completion

- Complication seen same time

- Cost effective

However not all setups have facility of radiologist available or physicians trained in ultrasound guidance. Also rising incidence of hypertension and diabetes and long survival has given rise to increased prevalence of patients having nephropathy and undergoing Hemodialysis. Poor compliance of patients and lack of access of hemodialysis centres results in patients getting admitted in pulmonary edema and severe metabolic acidosis in unstable hemodynamic condition here seeker needle method works better (Fig 2) Patient hemodynamic stability is needed for ultrasound method.

Some disadvantages of Ultrasound over landmark technique

- Requires radiologist or Training

- Need of USG machine near critical cases

- Shifting of patients as per machine availability

- Difficult in unstable cases

Seeker Needle some added benefits

- Easy to learn and gain confidence
- Exact depth of vein appreciated

- Reduced hematoma over direct method

- Emergency situation without radiological backup

- Reduced cost (if complications excluded)

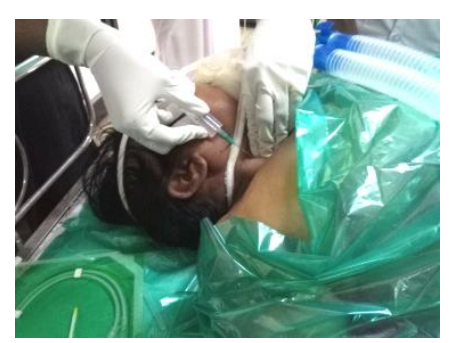

Fig 2. Seeker needle in Emergency

\section{Conclusions}

The Ultrasound guided HD canula insertion is the ideal method for inserting hemodialysis canula. It results in reduced complications. However in setups where easy Ultrasound machine or radiologist guidance is not possible, Seeker needle method in Landmark technique needed is an alternative and easy to learn, no shifting of machinery and can be done in peripheral setup where patient admitted in unstable hemodynamic conditions. Although this a optional method in inserting Hemodialysis canula, apart from one extra prick it offers lot of advantages over conventional method and places where immediate Ultrasound guidance not available.

\section{References}

1. McGee DC, Gould MK. Preventing complications of central venous catheterization. N Engl J Med 2003; 348:1123.

2. Ruesch S, Walder B, Tramer MR. Complications of central venous catheters: internal jugular versus subclavian access $-\mathrm{a}$ systematic review. Crit Care Med. 2002;30:454-460.

3. Bailey PL, Whitaker EE, Palmer LS, Glance LG. The accuracy of the central landmark used for central venous catheterization of the internal jugular vein.Anesth Analg 2006;102:1327-1332 
4. ZCJ Higgs, DAL Macafee, Seldinger technique 50 years on Lancet july 2005

5. Lefrant JY, Muller L, De La Coussaye JE, et al. Risk factors of failure and immediate complication of subclavian vein catheterization in critically ill patients. Intensive Care Med 2002; 28:1036.

6. Arrighi DA, Farnell MB, Mucha P Jr, et al. Prospective, randomized trial of rapid venous access for patients in hypovolemic shock. Ann Emerg Med 1989; 18:927.

7. Bailey PL, Glance LG, Eaton MP, Parshall B, McIntosh S. A survey of theuse of ultrasound during central venous catheterization. Anesth. Analg 2007;104(3):491-497

8. SELDINGER SI. Catheter replacement of the needle in percutaneous arteriography; a new technique. Acta radiol 1953; 39:368.

9. Ian Rigby, Daniel Howes, Jason Lord, Ian Walker Central venous acsess Kingston Resucitation institute

10. Jobes DR, Schwartz AJ, Greenhow DE, et al. Safer jugular vein cannulation: recognition of arterial puncture and preferential use of the external jugular route. Anesthesiology 1983; 59:353.

11. Albuquerque Júnior FC, Vasconcelos PR. Technical aspects of central venous catheterization. Curr Opin Clin Nutr Metab Care 1998; 1:297.

12. Jose Chako, Gagan Brar Bedside Ultrasonography IJCCM 2014 Vol 18 301-309

13. Denys BG, Uretsky BF. Anatomy variations of internal jugular vein location: impact of central venous access. Crit Care Med. 1991; 19:1516-9.

14. American College of Emergency Physicians. Emergency ultrasound guidelines. Ann EmergMed. 2009 Apr. 53(4):550-70.

15. Sznajder JI, Zveibil FR, Bitterman H, et al. Central vein catheterization. Failure and complication rates by three percutaneous approaches. Arch Intern Med 1986; 146:259. 